\title{
Ciclesonide Use in COVID-19: Not All Steroids Are the Same
}

\author{
Michel Tagliati ${ }^{1}$, Marianna Leopoulou ${ }^{2}$, Jo Ann LeQuang ${ }^{3}$, Charles E. Wollmuth ${ }^{3}$, \\ Joseph V. Pergolizzi ${ }^{3}$, Kailyn Mitchell ${ }^{3}$, Peter Magnusson ${ }^{4,5}$ \\ ${ }^{1}$ Proactive Health Inc., Stockholm, Sweden \\ ${ }^{2}$ Cardiology Clinic, Athens General Hospital “Elpis”, Athens, Greece \\ ${ }^{3}$ NEMA Research, Inc., Naples, Florida, USA \\ ${ }^{4}$ Centre for Research and Development, Region Gävleborg/Uppsala University, Gävle, Sweden \\ ${ }^{5}$ Department of Medicine, Cardiology Research Unit, Karolinska Institutet, Stockholm, Sweden \\ Email: ‘joannlequang@gmail.com
}

How to cite this paper: Tagliati, M., Leopoulou, M., LeQuang, J.A., Wollmuth, C.E., Pergolizzi, J.V., Mitchell, K. and Magnusson, P. (2021) Ciclesonide Use in COVID-19: Not All Steroids Are the Same. Pharmacology \& Pharmacy, 12, 10-24.

https://doi.org/10.4236/pp.2021.121002

Received: November 20, 2020

Accepted: January 26, 2021

Published: January 29, 2021

Copyright $\odot 2021$ by author(s) and Scientific Research Publishing Inc. This work is licensed under the Creative Commons Attribution International License (CC BY 4.0).

http://creativecommons.org/licenses/by/4.0/

\begin{abstract}
Introduction: The inflammatory mechanisms of COVID-19 suggest that corticosteroids may be beneficial, but their benefits must outweigh their potential risks. The RECOVERY trial results suggest that dexamethasone $6 \mathrm{mg} /$ day (but not other steroids) may confer mortality benefits on ventilated COVID-19 patients. Methods: This is a narrative review of the literature about the use of ciclesonide and dexamethasone for COVID-19 patients. Literature is being created rapidly and this review is offered as a state-of-the-science narration. Results: The SARS-CoV-2 virus is an RNA virus whose RNA is transcribed via open reading frames, making its elimination difficult. Coronaviruses have evolved multiple strategies for proteolytic activation of the spike; viral replication occurs entirely in the cytoplasm. In this connection, the RNA-cleaving endoribonuclease (NSP-15 also known as EndoU) may play a key role by facilitating viral double-stranded RNA recognition by the host's macrophages. Furthermore, the virus is able to undergo RNA recombination rapidly, enabling it to evade host immunity and develop drug resistance. Ciclesonide is an inhaled corticosteroid that reduces lung inflammation and blocks the activity of specific kinases which may explain its anti-inflammatory effect. Dexamethasone is known to reduce mortality in ventilated COVID-19 patients. Discussion: Systemic corticosteroids were used in previous coronavirus epidemics (SARS and MERS) and pulmonary histology of these patients is similar to those in COVID-19 patients. Acute respiratory distress syndrome is the main cause of death in most COVID-19 infections and steroids may be effective in addressing that condition, brought on by cytokine storm. However, it should be noted that inhaled steroids likely have a narrower window for effect than
\end{abstract}


systemic regimens. Conclusion: Dexamethasone has been proven to confer mortality benefits on ventilated COVID-19 patients and may be used with inhaled ciclesonide, which has few side effects and can be locally metabolized. Further study is needed.

\section{Keywords}

Ciclesonide, Coronavirus, COVID-19, Dexamethasone, SARS-CoV-2

\section{What Is Known and Objective}

The current pandemic due to the coronavirus disease 2019 (COVID-19) has spurred an effort to deploy the rational use or repurpose of approved registered pharmacological agents with potential efficacy. The inflammatory mechanisms of severe COVID-19 infection [1] [2] suggest that corticosteroids may be useful. However, there is evidence that argues against the routine systemic use of different types of corticosteroids, as the drug-associated side effects may often outweigh their potential benefits [3]. This is in line with previous recommendations from the World Health Organization (WHO) on the management of severe acute respiratory distress syndrome (ARDS) due to COVID-19, which advises against the use of steroids unless indicated for another reason [4]. Recent results from the UK-based RECOVERY trial emphasize the need to reevaluate this generalization, because it has been shown that dexamethasone treatment $6 \mathrm{mg} / \mathrm{day}$ significantly reduced 28-day mortality in patients with COVID-19 on ventilator support [5]. Notably, treatment arms with other steroids such as prednisolone, hydrocortisone, or methylprednisolone sodium succinate in the same study did not reduce mortality [5]. Nevertheless, there is much to be elucidated about the use of corticosteroids for COVID-19 patients in terms of specific agents, risks versus benefits, routes of administration, and mechanisms of action. Therefore, the purpose of this narrative review was to investigate the current knowledge with regard to mechanism and potential clinical rationale for further studies on inhaled versus oral steroids, especially with ciclesonide and dexamethasone.

\section{Methods}

A search in MEDLINE (English) using the terms "COVID-19", "inhalation steroids", "ciclesonide", and "dexamethasone" was conducted. Because the existing body of literature on the current pandemic is rapidly evolving, the authors also searched for relevant sources using Google Scholar, including preprints, until 16 July 2020 [6] [7]. The authors also implemented a broad dynamic approach and included information on aspects such as virology, molecular biology, immunology, genetics, biochemistry, pharmacology, pathophysiology, and clinical application to current date with special attention on ciclesonide and the latest clinical results using dexamethasone. 


\section{Results}

\subsection{About SARS-CoV-2}

Like polio, measles, Ebola, and flu viruses, the currently pandemic virus known as severe acute respiratory syndrome coronavirus-2 (SARS-CoV-2) is an RNA virus. Human strains of various coronaviruses are associated with approximately 15 percent of cases of the common cold [8]. In the 1970s and early 1980s, coronavirus virion proteins and nested-set arrangements of mRNAs were identified and the discontinuous nature of coronavirus transcription was initially demonstrated [9]. The first published sequence of a coronavirus gene appeared in 1983, starting an era of whole genome sequencing of four coronaviruses [10].

Coronaviruses have the largest RNA of all known RNA viruses. Its RNA is transcribed via open reading frames. This peculiarity increases the efficacy of viral replication and mutagenesis and makes it more difficult for the human immune system to eliminate [11]. The SARS-CoV-2 genome encodes 27 proteins needed by the virus to infect humans and to replicate [12]. These proteins include: the notorious spike protein, which recognizes angiotensin-converting enzyme 2 (ACE2) receptor in the initial stage of infection to gain intracellular access; two proteases, which cleave viral and human proteins; and the RNA polymerase (RdRp), which synthesizes viral RNA. The genome also contains a large replicase gene, encompassing nonstructural proteins (NSPs), followed by structural and accessory genes [13]. The S glycoprotein of coronaviruses, the main determinant of host cell attachment and viral entry, is not well conserved. Most human coronaviruses use different host cell receptors for viral entry and may also require different host cell proteases that allow fusion of viral and cellular membranes [14].

Coronaviruses have evolved multiple strategies for proteolytic activation of the spike, and a large number of host proteases have been shown to proteolytically process the spike protein. These include, but are not limited to, endosomal cathepsins, cell surface transmembrane protease/serine proteases, furin, and trypsin [15]. The most conserved proteins among coronaviruses are NSPs involved in essential functions of the viral lifecycle [16]. Coronavirus replication takes place entirely in the cytoplasm, and it is believed that nuclear functions are not required for RNA synthesis [17]. Thus, viral RNA transcription does not involve the conventional RNA splicing machinery present in the nucleus.

The structural proteins that make up the virion are less conserved than NSPs, and accessory proteins are only functionally conserved among very closely related viruses [18]. While several functions of NSPs have been linked to RNA replication and processing of RNA, the roles of other proteins are poorly understood or remain unknown [19]. The NSPs perform essential functions in immune antagonism through formation of the viral replication complex and double membrane vesicles, which shield the viral RNA, viral RNA proofreading, binding of nucleic acid, helicase activity, and so on [20]. Another essential element of the virus lifecycle is proteolytic processing of viral polyproteins into functional 
NSPs by two viral proteases, 3CLpro and PLpro. Specifically, 3CLpro, also known as NSP5, is first automatically cleaved from polyproteins to produce mature enzymes, and then further cleaves downstream NSPs at 11 sites to release NSP14-NSP16. The 3CLpro directly mediates the maturation of NSPs, which is essential in the life cycle of the virus [21].

In addition to polymerase and protease functions, other essential functions performed by the NSPs of the coronavirus include immune antagonism, double membrane vesicle organization, scaffolding for replication complex formation, nucleic acid binding, helicase activity, and viral RNA proofreading, which may be future targets of coronavirus specific antiviral drug discovery [22].

\subsection{NSP-15 and Its Role in Coronavirus Biology}

Of particular interest in this context is one NSP in particular, the RNA-cleaving endoribonuclease (NSP15). The NSP15-associated endoribonuclease (EndoU) domain is one of the most conserved proteins among corona- and related viruses. NSP15 is also referred to as coronavirus EndoU in the scientific literature. However, the role of NSP15 in coronavirus replication was enigmatic, as EndoUdeficient coronaviruses were viable and replicated to near wild-type virus levels in fibroblast cells. Nevertheless, NSP15 is essential in coronavirus biology [23]-[28]. A breakthrough in the understanding of the role of EndoU was demonstrated in recent studies, which showed that EndoU mediates the evasion of viral double-stranded RNA (dsRNA) recognition by host sensors in macrophages [29]. There are also suggestions that NSP15 degrades viral RNA to hide it from the host defenses. The loss of NSP15 activity also resulted in greatly attenuated disease in mice and stimulated a protective immune response. Taken together, these findings demonstrate that coronavirus NSPs are critical for the evasion of host dsRNA sensors in macrophages [30].

\subsection{SARS-Cov-2 Mutations}

RNA viruses are characterized by a high mutation rate, up to a million times higher than that of their hosts, and SARS-CoV-2 is no exception [31]. It is also important to recognize whether the presence of some mutations might correlate with different SARS-CoV-2 mortality rates. Viral mutagenic capability depends upon several factors, including the fidelity of viral enzymes that replicate nucleic acids, such as SARS-CoV-2 RNA dependent RdRP [32]. Thus, recombination is a well-characterized feature of coronavirus biology. It can potentially provide a genetic mechanism by which coronaviruses maintain their sequence integrity. In light of the large size of the coronavirus RNA, it is predictable that most of the viral RNA molecules would contain one or more mutations, due to the high error frequencies of RNA polymerases; homologous recombination repair may provide a mechanism for the virus to stabilize their genome and increase fidelity across generations.

One unique genetic feature of coronaviruses is their ability to undergo RNA 
recombination at a very high frequency [33]. Mutation rate drives viral evolution and genome variability, thereby enabling viruses to escape host immunity and to develop drug resistance [34] [35]. Based on genomic sampling over time, the substitution rate is estimated to be 0.00084 per site per year as of 16 May 2020, two- to six-fold lower than the substitution rate for influenza $(0.004-0.005$ subst/site/yr for influenza A and for influenza B [36]. Across its $\approx 30,000$ basepair genome, the SARS-CoV-2 thus undergoes roughly one genetic change every other week. To date, over 14,000 mutations have been identified [37].

These findings suggest that the SARS-Cov-2 virus is evolving, and that unique European, North American, and Asian strains might coexist, each with a different mutation pattern. A SARS-CoV-2 variant carrying the spike protein amino acid change D614G has become the most prevalent form in the global pandemic. Dynamic tracking of variant frequencies revealed a recurrent pattern of G614 increase at multiple geographic levels: national, regional, and municipal. The shift occurred even in local epidemics where the original D614 form was well established prior to the introduction of the G614 variant. The consistency of this pattern suggests that the G614 variant may have a fitness advantage [38].

Since RNA viruses need their RdRP for replication, this means that, in theory, the COVID-19 pandemic could be treated effectively by a series of RdRP inhibitors. However, adenosintriphosphate (ATP) antagonists in general could potentially inhibit many other host enzymes, such as ATPases, protein kinases, and chaperones, and could therefore cause a number of side effects, in the same many as chemotherapeutic agents [39]. Indeed, the RECOVERY-trail treatment arms for ATP-antagonists, such as remdesivir, showed no significant reduction of mortality [40]. For treating COVID-19 in humans, it would be necessary to find far more selective pharmacological agents, namely one which targets a specific host enzyme that is essential for viral infection but not crucial to normal physiology of the host. In human cells, PAK1 RAC/CDC42-activated kinase is the major pathogenic kinase, the abnormal activation of which has been associated with everything from cancer, malaria, and pandemic viral infections to inflammation, immunosuppression, and accelerated aging symptoms [41].

\subsection{Ciclesonide and Its Mechanism of Action}

Ciclesonide was patented and approved in 2008 in the United States for the treatment of inflammatory lung disease, such as asthma. It is an inhaled corticosteroid that reduces lung inflammation and has been approved for the treatment of asthma and respiratory symptoms. Because of its use in reducing pulmonary inflammation, ciclesonide is of special interest in the context of COVID-19. In order to understand the postulated ciclesonide inhibitory action on COVID-19 in infected human cells, mammalian family kinases called PAKs (RAC/CDC42-activated kinases) which were originally cloned more than 25 years ago were recently further studied [42] [43].

Caffeic acid and its ester, caffeic phenethyl ester occur in propolis (a natural 
substance produced by honeybees), the first natural ingredient shown to inhibit RAC and act as a PAK1-blocker [44]. But there are other anti-viral effects in other drugs. The anti-viral effect of remdesivir $\left(\mathrm{IC}_{50}\right.$ around $1 \mu \mathrm{M}$ of each) was recently confirmed in vitro [45]. Furthermore, the expression of LLC2 depends on the coronavirus ACE2-induced CK2/RAS-PAK1-RAF-AP1 signaling pathways. Taken altogether, these observations clearly indicate the PAK1-dependency of coronaviral pathogenesis, and strongly suggest, if not proven clinically as yet, that PAK1-blockers in general might be useful for the treatment of COVID-19. Ciclesonide most probably exerts its anti-inflammatory effect by blocking PAK1 [46].

In order to elucidate the mechanism of action of ciclesonide, glucocorticoids and their recent and current use in clinical practice were reviewed. Glucocorticoids are synthesized in the adrenal cortex and secreted into the blood, where the levels of glucocorticoids fluctuate in a circadian mode [47]. In humans, the naturally occurring glucocorticoid is hydrocortisone (cortisol), which is synthesized from its precursor cortisone. The beneficial effects of glucocorticoids in asthma were first described in 1950. Treatment with glucocorticoids has been consistently shown not only to relieve asthma symptoms, but also enhance bronchial hyperresponsiveness [48]. Corticosteroids (methylprednisolone) were given as treatment during the SARS and Middle East Respiratory Syndrome (MERS) epidemics due to their immunomodulatory effects, which suppress inflammatory responses, but the result of their use showed no perceived benefit and possible deleterious effects [49] [50]. These effects may be mediated in part by modulation of epithelial cell functions, since many in vitro and in vivo studies, have shown that glucocorticoids are able to modulate the inflammatory functions of bronchial epithelial cells.

Glucocorticoids inhibit the expression of a large number of inflammation-associated molecules, including cytokines, chemokines, arachidonic acid metabolites, and adhesion molecules [51]. These effects predominantly are mediated via the inhibition of NF- $\mathrm{kB}$ activity. In contrast, anti-inflammatory mediators, such as NEP and IL-1 receptor antagonists, often are up-regulated by glucocorticoids. The beneficial effects of glucocorticoid in asthma have been demonstrated by in vivo studies showing that treatment of asthmatic patients with inhaled glucocorticoids inhibits the airway inflammation and simultaneously improves lung function [52]. Favorable modulation of the immune response is considered one of the possible mechanisms by which corticosteroids might be beneficial or lead to improved clinical outcomes in the treatment of severe acute respiratory coronavirus infections, including COVID-19, SARS, and MERS.

Common to severe cases of coronavirus infections is the presence of hypercytokinemia (also called cytokine storm) and development of acute lung injury or ARDS. Pathologically, diffuse alveolar damage has been found on autopsy [53]. A growing volume of clinical trial data from patients with severe community-acquired pneumonia, ARDS, or septic shock suggest benefit from low-to 
moderate dose corticosteroids with respect to mortality and length of stay [54] [55] [56]. A systematic review of (mainly low-dose) corticosteroid trials in severe sepsis and septic shock did not identify any increased risk of gastroduodenal bleeding, superinfection, or neuromuscular weakness; however, an association with an increased risk of hyperglycemia and hypernatremia was noted [57] [58].

Ciclesonide is a parent compound that is converted locally in airways by esterases to produce the active metabolite, desisobutyryl-ciclesonide (des-CIC) [59]. The active metabolite, des-CIC, has a 100-fold greater relative glucocorticoid receptor binding affinity than ciclesonide itself (relative glucocorticoid receptor binding affinities are 1200 and 12, respectively; dexamethasone reference is 100) [60]. If any ciclesonide enters the circulation, it is highly protein bound (99\%) and extensively metabolized by liver oxidases, resulting in very low systemic exposure [61]. Ciclesonide is delivered in solution form via a hydrofluoroalkane metered-dose inhaler with a once-daily dosing schedule, which facilitates patient compliance. Clinical studies demonstrate that ciclesonide is as effective as existing gold standard inhaled corticosteroids for the control of asthma and has a good safety profile [62]. At its target sites, the lungs, ciclesonide is converted to the active metabolite des-CIC, in a process that is called on-site activation. Pharmacokinetically, high lipophilicity and the formation of a local depot prolong the pulmonary duration of action for des-CIC, explaining the once-daily administration of ciclesonide in clinical practice [63].

Ciclesonide exerts its anti-inflammatory effect most probably by blocking PAK1. Ciclesonide has been shown to block PAK1-dependent replication and pathogenesis in vitro [64] Ciclesonide blocks SARS-CoV-2 RNA replication in vitro and inhibits cytopathic activity. Ciclesonide demonstrates low cytotoxicity and potent suppression of MERS viral growth. The most commonly used orally administered steroids, cortisone, prednisolone, and dexamethasone do not suppress viral growth, nor did the commonly used inhaled steroid fluticasone. The effective concentration of ciclesonide to block SARS-CoV-2 replication (EC90) was $6.3 \mu \mathrm{M}$; ciclesonide $10 \mathrm{mg} / \mathrm{kg} /$ day exerts strong suppression of PAK1-dependent growth of lung cancer (A541 cell line) [65]. In addition to its known anti-inflammatory properties, ciclesonide seems to be able to block coronavirus RNA replication [66]. Ciclesonide is used to treat inflammatory diseases, such as asthma and allergic rhinitis [67] [68].

\section{Discussion}

Systemic corticosteroids were widely used during previous coronavirus outbreaks (SARS and MERS); however, a systematic review of published literature on their use in SARS did not conclude that treatment was beneficial and found some evidence of possible harm [69] [70] [71] [72]. At present, there is no evidence as to whether pre-morbid use or continued administration of inhaled corticosteroids is a factor for adverse or beneficial outcomes in acute respiratory in- 
fections due to coronavirus [73] [74] [75]. Schaller et al. performed post mortem examinations on 10 patients with verified COVID-19 and found that in 9 patients, infiltrations with ground-glass opacity predominantly in the middle and lower lung fields were detected by chest X-ray, and organized diffuse alveolar damage and SARS-CoV-2 persistence in the respiratory tract were the predominant histopathologic findings and constituted the leading cause of death in patients with and without invasive ventilation [76]. The pulmonary histologic characteristics of COVID-19 resembled those observed in diseases caused by other beta-coronavirus infections such as SARS and MERS [77].

Further data and studies are urgently needed. As a start, it is essential that epidemiological studies of COVID-19 include detailed information on patients' comorbidities and prior medications in order to allow analyses of possible benefits or harms of inhaled corticoid steroids or other therapies [78]. In a recent study, Gralinsky et al. [79] demonstrated that complement activation is related to more severe respiratory consequences in SARS coronavirus infections, another hint of the importance of understanding the cascade of events that enhance damage to the host in infections with novel coronaviruses. Most evidence to date show that ARDS is the main cause of death in COVID-19 and that ARDS is the common immunopathological event for SARS-CoV-2 infections, and its predecessors, SARS-CoV and MERS-CoV infections [80] [81]. One of the main mechanisms for ARDS is the cytokine storm, the deadly uncontrolled systemic inflammatory response resulting from the release of large amounts of different pro-inflammatory cytokines; interferons, interleukins, tumor necrosis factor- $\alpha$, transforming growth factor- $\beta$ and chemokines [82] [83] [84] [85]. The suggestion by Mehta to consider active immunosuppression as a strategic tool leads to the question as to the appropriate deployment of ciclesonide, for example, whether inhaled ciclesonide should be used early in the disease (and at what dose) and replaced by oral steroids later. However, the window in which oral steroids might be beneficial to patients with COVID-19 is likely narrow compared to that of inhalation steroids, which have documented effect in vitro studies.

\section{What Is New and Objective}

Secondary complications of COVID-19, such as bacterial pneumonia, may require antibiotics [86]. Patients treated with inhalation steroids are at increased risk for bacterial pneumonia [87]. In COVID-19, patient stratification by risk of disease severity has eluded us with the result that hospitals emphasize treatment of the critically ill, while it may be more beneficial in this disease to intervene early. The liberal use of a two-week course of prescribed inhalation ciclesonide as a prophylactic for outpatients with COVID-19 symptoms should be considered. Of course, there is still a risk that some of these outpatients may develop severe respiratory symptoms [88]. But this approach is the gold standard for patients with respiratory syncytial virus infections [89], COPD, and asthma ex- 
acerbation [90]. There is also an urgent need to assess the benefits of inhaled corticosteroids for treating COVID-19 patient with and without underlying chronic respiratory disease. Further studies on inhaled steroids would be beneficial and are ongoing [82] [83] [84].

Many of the treatments used in SARS or MERS patients in outbreak situations were not based on clear in vitro and in vivo model evidence of efficacy, and meta-analyses of treatments failed to show effective therapeutic regimens. In clinical praxis it is routine to treat severe asthma with increased doses of steroids and these recommendations remain unchanged during the pandemic. There have been observations of increased mortality in exacerbations of chronic pulmonary obstructive disease (COPD) in these groups when analyzing COVID-19 mortality data, indicating that the inhalation steroids contribute to increased mortality, according to some authors [69]. Surprisingly, the prevalence of COPD among patients with SARS and COVID-19 appears to be lower than among the general population [70].

The co-occurrence of asthma with obesity, another predictor of poor outcome in COVID-19 patients, places obese patients with asthma at markedly higher risk for worse outcomes [71]. Inhaled corticosteroids use undoubtedly reduces the rate of exacerbations in both asthma and COPD. If people with stable asthma stop or reduce their inhaled corticosteroids inappropriately in response to concerns about immunosuppression and worries about developing COVID-19, they may be at significant risk of having an exacerbation [72]. Approximately $40 \%$ $60 \%$ of COPD and up to $80 \%$ of asthma exacerbations occur due to viral infections, including common coronaviruses [73]. Approximately 25\% of patients admitted to the hospital developed ARDS a median of 10.5 days after symptom onset [86]. Sadly, patients with underlying lung disease hospitalized with COVID-19 have worse outcomes [87] [88] [89]. In people with COPD, inhaled corticosteroid use is associated with a higher prevalence of pneumonia and a change in the lung microbiome, although not a change in respiratory virus detection [90].

\section{Conclusion}

Dexamethasone reduces mortality in severe COVID-19 patients requiring ventilator treatment. Less is known about steroid treatment in milder forms of COVID-19. Ciclesonide is a safe and effective inhaled steroid with few side effects. As ciclesonide is inhaled, it is metabolized locally in the most affected critical organ of infected patients. Its metabolites have far higher receptor affinity than that of dexamethasone, so it is reasonable to assume a greater clinical effect. Therefore, randomized controlled trials are welcome to elucidate the role of inhaled corticosteroids in COVID-19 in addition to their use in patients with preexisting conditions.

\section{Acknowledgements}

The authors received no funding in support of this article. 


\section{Conflicts of Interest}

The authors declare no conflicts of interest regarding the publication of this paper.

\section{References}

[1] Ye, Q., Wang, B. and Mao, J. (2020) The Pathogenesis and Treatment of the "Cytokine Storm" in COVID-19. Journal of Infection, 80, 607-613. https://doi.org/10.1016/j.jinf.2020.03.037

[2] AminJafari, A. and Ghasemi, S. (2020) The Possible of Immunotherapy for Covid-19: A Systematic Review. International Immunopharmacology, 83, Article ID: 106455. https://doi.org/10.1016/j.intimp.2020.106455

[3] Veronese, N., Demurtas, J., Yang, L., et al. (2020) Use of Corticosteroids in Coronavirus Disease 2019 Pneumonia: A Systematic Review of the Literature. Frontiers in Medicine, 7, 170. https://doi.org/10.3389/fmed.2020.00170

[4] WHO (2020) Clinical Management of Severe Acute Respiratory Infection (SARI) When COVID-19 Disease Is Suspected.

https://www.who.int/docs/default-source/coronaviruse/clinical-management-of-no vel-cov.pdf

[5] Dexamethasone for COVID-19-Preliminary Report Effect of Dexamethasone in Hospitalized Patients with COVID-19-Preliminary Report RECOVERY Collaborative Group.

[6] Shultz, M. (2007) Comparing Test Searches in PubMed and Google Scholar. Journal of the Medical Library Association, 95, 442-445.

https://doi.org/10.3163/1536-5050.95.4.442

[7] Henderson, J. (2005) Google Scholar: A Source for Clinicians? CMAJ, 172, 1549-1550. https://doi.org/10.1503/cmaj.050404

[8] Mesel-Lemoine, M., Millet, J., Vidalain, P.-O., et al. (2012) A Human Coronavirus Responsible for the Common Cold Massively Kills Dendritic Cells but Not Monocytes. Journal of Virology, 86, 7577-7587. https://doi.org/10.1128/JVI.00269-12

[9] Masters, P.S. (2006) The Molecular Biology of Coronaviruses. Advances in Virus Research, 65, 193-292. https://doi.org/10.1016/S0065-3527(06)66005-3

[10] Wang, H., Li, X., Li, T., et al. (2020) The Genetic Sequence, Origin, and Diagnosis of SARS-CoV-2. European Journal of Clinical Microbiology and Infectious Diseases, 39, 1629-1635. https://doi.org/10.1007/s10096-020-03899-4

[11] Kuljić-Kapulica, N. and Budisin, A. (1992) Coronaviruses. Srpski Arhiv za Celokupno Lekarstvo, 120, 215-218.

[12] Stancioiu, F., Papadakis, G.Z., Kteniadakis, S., Izotov, B.N., Coleman, M.D., Spandidos, D.A. and Tsatsakis, A. (2020) A Dissection of SARS-CoV2 with Clinical Implications (Review). International Journal of Molecular Medicine, 489-508.

https://doi.org/10.3892/ijmm.2020.4636 https://www.spandidos-publications.com/ijmm/46/2/489

[13] Ziebuhr, J. (2004) Molecular Biology of Severe Acute Respiratory Syndrome Coronavirus. Current Opinion in Microbiology, 7, 412-419. https://doi.org/10.1016/j.mib.2004.06.007

[14] Totura, A.L. and Bavari, S. (2019) Broad-Spectrum Coronavirus Antiviral Drug Discovery. Expert Opinion on Drug Discovery, 14, 397-412.

https://doi.org/10.1080/17460441.2019.1581171 
[15] Millet, J.K. and Whittaker, G.R. (2015) Host Cell Proteases: Critical Determinants of Coronavirus Tropism and Pathogenesis. Virus Research, 202, 120-134. https://doi.org/10.1016/j.virusres.2014.11.021

[16] Snijder, E.J., Decroly, E. and Zeibuhr, J. (2016) The Nonstrucutral Proteins Directing Coronavirus RNA Synthesis and Processing. In: Advances in Virus Research, Vol. 96, Academic Press, Cambridge, MA, 59-126. https://doi.org/10.1016/bs.aivir.2016.08.008

[17] Hagemeijer, M.C., Verheije, M.H., Ulasli, M., et al. (2010) Dynamics of Coronavirus Replication-Transcription Complexes. Journal of Virology, 84, 2134-2149. https://doi.org/10.1128/JVI.01716-09

[18] Mousavizadeh, L. and Ghasemi, S. (2020) Genotype and Phenotype of COVID-19: Their Roles in Pathogenesis. Journal of Microbiology, Immunology and Infection, in press. https://doi.org/10.1016/j.jmii.2020.03.022

[19] Kim, Y., Jedrzejczak, R., Maltseva, N.I., et al. (2020) Crystal Structure of Nsp15 Endoribonuclease NendoU from SARS-CoV-2. Protein Science, 29, 1596-1605. https://doi.org/10.1002/pro.3873

[20] Fehr, A.R. and Perlman, S. (2015) Coronaviruses: An Overview of Their Replication and Pathogenesis. In: Maier, H., Bickerton, E. and Britton, P. (Eds.), Coronaviruses: Methods and Protocols, Springer, New York, 1-23. https://doi.org/10.1007/978-1-4939-2438-7_1

[21] Wu, C., Liu, Y., Yang, Y., et al. (2020) Analysis of Therapeutic Targets for SARSCoV-2 and Discovery of Potential Drugs by Computational Methods. Acta Pharmaceutica Sinica B, 10, 766-788. https://doi.org/10.1016/j.apsb.2020.02.008

[22] Neuman, B.W., Chamberlain, P., Bowden, F. and Joseph, J. (2014) Atlas of Coronavirus Replicase Structure. Virus Research, 194, 49-66. https://doi.org/10.1016/j.virusres.2013.12.004

[23] Guarino, L.A., Bhardwaj, K., Dong, W., Sun, J., Holzenburg, A. and Kao, C. (2005) Mutational Analysis of the SARS Virus Nsp15 Endoribonuclease: Identification of Residues Affecting Hexamer Formation. Journal of Molecular Biology, 353, 1106-1117. https://doi.org/10.1016/j.jmb.2005.09.007

[24] Zhang, L., Li, L., Yan, L., et al. (2018) Structural and Biochemical Characterization of Endoribonuclease Nsp15 Encoded by Middle East Respiratory Syndrome Coronavirus. Journal of Virology, 92, e00893-18.

[25] Joseph, J.S., Saikatendu, K.S., Subramanian, V., et al. (2007) Crystal Structure of a Monomeric Form of Severe Acute Respiratory Syndrome Coronavirus Endonuclease nsp15 Suggests a Role for Hexamerization as an Allosteric Switch. Journal of Virology, 81, 6700-3708. https://doi.org/10.1128/JVI.02817-06

[26] Bhardwaj, K., Sun, J., Holzenburg, A., Guarino, L.A. and Kao, C.C. (2006) RNA Recognition and Cleavage by the SARS Coronavirus Endoribonuclease. Journal of Molecular Biology, 361, 243-256. https://doi.org/10.1016/j.jmb.2006.06.021

[27] Bhardwaj, K., Guarino, L. and Kao, C.C. (2004) The Severe Acute Respiratory Syndrome Coronavirus Nsp15 Protein Is an Endoribonuclease That Prefers Manganese as a Cofactor. Journal of Virology, 78, 12218-12224. https://doi.org/10.1128/JVI.78.22.12218-12224.2004

[28] Deng, X. and Baker, S.C. (2018) An “Old” Protein with a New Story: Coronavirus Endoribonuclease Is Important for Evading Host Antiviral Defenses. Virology, 517, 157-163. https://doi.org/10.1016/j.virol.2017.12.024

[29] Deng, X., Hackbart, M., Mettelman, R.C., et al. (2017) Coronavirus Nonstructural Protein 15 Mediates Evasion of dsRNA Sensors and Limits Apoptosis in Macro- 
phages. Proceedings of the National Academy of Sciences of the United States of America, 114, E4251-E4260. https://doi.org/10.1073/pnas.1618310114

[30] Duffy, S. (2018) Why Are RNA Virus Mutation Rates So Damn High? PLoS Biology, 16, E4251-E4260. https://doi.org/10.1371/journal.pbio.3000003

[31] Pachetti, M., Marini, B., Benedetti, F., et al. (2020) Emerging SARS-CoV-2 Mutation Hot Spots Include a Novel RNA-Dependent-RNA Polymerase Variant. Journal of Translational Medicine, 18, Article No. 179. https://doi.org/10.1186/s12967-020-02344-6

[32] Sanjuán, R. and Domingo-Calap, P. (2016) Mechanisms of Viral Mutation. Cellular and Molecular Life Sciences, 73, 4433-4448. https://doi.org/10.1007/s00018-016-2299-6

[33] Regoes, R.R., Hamblin, S. and Tanaka, M.M. (2013) Viral Mutation Rates: Modelling the Roles of Within-Host Viral Dynamics and the Trade-Off between Replication Fidelity and Speed. Proceedings of the Royal Society B: Biological Sciences, 280, Article ID: 20122047. https://doi.org/10.1098/rspb.2012.2047

[34] Eckerle, L.D., Becker, M.M., Halpin, R.A., et al. (2010) Infidelity of SARS-CoV Nsp14-Exonuclease Mutant Virus Replication Is Revealed by Complete Genome Sequencing. PLoS Pathogens, 6, e1000896. https://doi.org/10.1371/journal.ppat.1000896

[35] Hadfield, J., Megill, C., Bell, S.M., et al. (2018) NextStrain: Real-Time Tracking of Pathogen Evolution. Bioinformatics, 34, 4121-4123.

https://doi.org/10.1093/bioinformatics/bty407

[36] Day, T., Gandon, S., Lion, S. and Otto, S.P. (2020) On the Evolutionary Epidemiology of SARS-CoV-2. Current Biology, 30, R849-R857. https://doi.org/10.1016/j.cub.2020.06.031

[37] Korber, B., Fischer, W.M., Gnanakaran, S., et al. (2020) Tracking Changes in SARS-CoV-2 Spike: Evidence That D614G Increases Infectivity of the COVID-19 Virus. Cell, 182, 812-827.E19. https://doi.org/10.1016/j.cell.2020.06.043

[38] Yin, W., Mao, C., Luan, X., et al. (2020) Structural Basis for Inhibition of the RNA-Dependent RNA Polymerase from SARS-CoV-2 by Remdesivir. Science, $\mathbf{3 6 8}$, 1499-1504. https://pubmed.ncbi.nlm.nih.gov/32358203/

[39] RECOVERY and RemdesivirV1.0 2020-05-27. https://www.recoverytrial.net/

[40] Maruta, H. (2014) Herbal Therapeutics That Block the Oncogenic Kinase PAK1: A Practical Approach towards PAK1-Dependent Diseases and Longevity. Phytotherapy Research, 28, 656-672. https://doi.org/10.1002/ptr.5054

[41] Zhao, Z. and Manser, E. (2012) PAK Family Kinases. Cellular Logistics, 2, 59-68. https://doi.org/10.4161/cl.21912

[42] Maruta, H. and He, H. (2020) PAK1-Blockers: Potential Therapeutics against COVID-19. Medicine in Drug Discovery, 6, Article ID: 100039. https://doi.org/10.1016/j.medidd.2020.100039

[43] Demestre, M., Messerli, S.M., Celli, N., et al. (2009) CAPE (Caffeic Acid Phenethyl Ester)-Based Propolis Extract (Bio 30) Suppresses the Growth of Human Neurofibromatosis (NF) Tumor Xenografts in Mice. Phytotherapy Research, 23, 226-230. https://doi.org/10.1002/ptr.2594

[44] Wang, M., Cao, R., Zhang, L., et al. (2020) Remdesivir and Chloroquine Effectively Inhibit the Recently Emerged Novel Coronavirus (2019-nCoV) in Vitro. Cell Research, 30, 269-271. https://doi.org/10.1038/s41422-020-0282-0

[45] Chen, I.-Y., Chang, S.C., Wu, H.-Y., et al. (2010) Upregulation of the Chemokine (C-C 
Motif) Ligand 2 via a Severe Acute Respiratory Syndrome Coronavirus Spike-ACE2 Signaling Pathway. Journal of Virology, 84, 7703-7712. https://doi.org/10.1128/JVI.02560-09

[46] Matsuyama, S., Kawase, M., Nao, N., et al. (2020) The Inhaled Corticosteroid Ciclesonide Blocks Coronavirus RNA Replication by Targeting Viral NSP15.

[47] Fuller, P.J., Lim-Tio, S.S. and Brennan, F.E. (2000) Specificity in Mineralocorticoid versus Glucocorticoid Action. Kidney International, 57, 1256-1264. https://doi.org/10.1046/j.1523-1755.2000.00959.x

[48] Stockman, L.J., Bellamy, R. and Garner, P. (2017) SARS: Systematic Review of Treatment Effects. PLoS ONE, 12, e343.

[49] Ramadan, A.A., Gaffin, J.M., Israel, E. and Phipatanakul, W. (2019) Asthma and Corticosteroid Responses in Childhood and Adult Asthma. Clinics in Chest Medicine, 40, 163-177. https://doi.org/10.1016/j.ccm.2018.10.010

[50] Morra, M.E., van Thanh, L., Kamel, M.G., et al. (2018) Clinical Outcomes of Current Medical Approaches for Middle East Respiratory Syndrome: A Systematic Review and Meta-Analysis. Reviews in Medical Virology, 28, e1977.

https://doi.org/10.1002/rmv.1977

[51] van der Velden, V.H.J. (1998) Glucocorticoids: Mechanisms of Action and Anti-Inflammatory Potential in Asthma. Mediators in Inflammation, 7, Article ID: 702547. https://doi.org/10.1080/09629359890910

[52] Barnes, P.J. (2010) Inhaled Corticosteroids. Pharmaceuticals, 3, 514-540. https://doi.org/10.3390/ph3030514

[53] Zhang, W., Zhao, Y., Zhang, F., et al. (2020) The Use of Anti-Inflammatory Drugs in the Treatment of People with Severe Coronavirus Disease 2019 (COVID-19): The Experience of Clinical Immunologists from China. Clinical Immunology, 214, Article ID: 108393. https://doi.org/10.1016/j.clim.2020.108393

[54] Xu, Z., Shi, L., Wang, Y., et al. (2020) Pathological Findings of COVID-19 Associated with Acute Respiratory Distress Syndrome. The Lancet Respiratory Medicine, 8, 420-422. https://doi.org/10.1016/S2213-2600(20)30076-X

[55] Rochwerg, B., Oczkowski, S.J., Siemieniuk, R.A.C., et al. (2018) Corticosteroids in Sepsis: An Updated Systematic Review and Meta-Analysis. Critical Care Medicine, 46, 1411-1420. https://doi.org/10.1097/CCM.0000000000003262

[56] Villar, J., Ferrando, C., Martínez, D., et al. (2020) Dexamethasone Treatment for the Acute Respiratory Distress Syndrome: A Multicentre, Randomised Controlled Trial. The Lancet Respiratory Medicine, 8, 267-276. https://doi.org/10.1016/S2213-2600(19)30417-5

[57] Hardeman, H., Grutters, J.C., van de Garde, M.W., et al. (2011) Dexamethasone and Length of Hospital Stay in Patients with Community-Acquired Pneumonia: A Randomised, Double-Blind, Placebo-Controlled Trial. The Lancet, 377, 2023-2053. https://doi.org/10.1016/S0140-6736(11)60607-7

[58] Annane, D., Bellissant, E., Bollaert, P.-E., et al. (2009) Corticosteroids in the Treatment of Severe Sepsis and Septic Shock in Adults: A Systematic Review. JAMA, 301, 2362-2375.http://www.bireme.br

[59] Stein, S.W. and Thiel, C.G. (2017) The History of Therapeutic Aerosols: A Chronological Review. Journal of Aerosol Medicine and Pulmonary Drug Delivery, 30, 20-41. https://doi.org/10.1089/jamp.2016.1297

[60] Christie, P. (2004) Ciclesonide: A Novel Inhaled Corticosteroid for Asthma. Drugs of Today, 40, 569-576. 
https://journals.prous.com/journals/servlet/xmlxsl/pk_journals.xml_summary_pr?p JournalId=4\&p_RefId=850475\&p_IsPs $=\mathrm{N}$

[61] Nave, R., Bethke, T.D., van Marle, S.P. and Zech, K. (2004) Pharmacokinetics of $\left[{ }^{14} \mathrm{C}\right]$ Ciclesonide after Oral and Intravenous Administration to Healthy Subjects. Clinical Pharmacokinetics, 43, 479-486. https://doi.org/10.2165/00003088-200443070-00004

[62] Nave, R., Watz, H., Hoffmann, H., Boss, H. and Magnussen, H. (2010) Deposition and Metabolism of Inhaled Ciclesonide in the Human Lung. European Respiratory Journal, 36, 1113-1119. https://doi.org/10.1183/09031936.00172309

[63] Ukena, D. (2005) Ciclesonid-Ein neues inhalatives kortikosteroid. Pneumologie, 59, 689-695. https://doi.org/10.1055/s-2005-915570

[64] Jeon, S., Ko, M., Lee, J., et al. (2020) Identification of Antiviral Drug Candidates against SARS-CoV-2 from FDA-Approved Drugs. Antimicrobial Agents and Chemotherapy, 64, e00819-20.

[65] Choi, H.S., Kim, S.L., Kim, J.H. and Lee, D.S. (2020) The FDA-Approved Anti-Asthma Medicine Ciclesonide Inhibits Lung Cancer Stem Cells through Hedgehog Signaling-Mediated SOX2 Regulation. International Journal of Molecular Sciences, 21, 1014. https://doi.org/10.3390/ijms21031014

[66] Allen, J.D., Jaffer, Z.M., Park, S.J., et al. (2009) P21-Activated Kinase Regulates Mast Cell Degranulation via Effects on Calcium Mobilization and Cytoskeletal Dynamics. Blood, 113, 2695-2705. https://doi.org/10.1182/blood-2008-06-160861

[67] Vogelmeier, C.F., Hering, T., Lewin, T., Sander, P. and Bethke, T.D. (2011) Efficacy and Safety of Ciclesonide in the Treatment of 24,037 Asthmatic Patients in Routine Medical Care. Respiratory Medicine, 105, 186-194.

https://doi.org/10.1016/j.rmed.2010.09.016

[68] Derendorf, H. (2007) Therapeutic Review: Pharmacokinetic and Pharmacodynamic Properties of Inhaled Ciclesonide. Journal of Clinical Pharmacology, 47, 782-789. https://doi.org/10.1177/0091270007299763

[69] Halpin, D.M.G., Faner, R., Sibila, O., Badia, J.R. and Agusti, A. (2020) Do Chronic Respiratory Diseases or Their Treatment Affect the Risk of SARS-CoV-2 Infection? The Lancet Respiratory Medicine, 8, 436-438.

https://doi.org/10.1016/S2213-2600(20)30167-3

[70] Mahdavinia, M., Foster, K.J., Jauregui, E., et al. (2020) Asthma Prolongs Intubation in COVID-19. The Journal of Allergy and Clinical Immunology. In Practice, 8, 2388-2391. https://www.ncbi.nlm.nih.gov/pmc/articles/PMC7224651/

[71] Johnston, S.L. (2005) Overview of Virus-Induced Airway Disease. Proceedings of the American Thoracic Society, 2, 150-156.

[72] Wu, C., Chen, X., Cai, Y., et al. (2020) Risk Factors Associated with Acute Respiratory Distress Syndrome and Death in Patients with Coronavirus Disease 2019 Pneumonia in Wuhan, China. JAMA Internal Medicine, 180, 934-943. https://doi.org/10.1001/jamainternmed.2020.0994

[73] Kurai, D., Saraya, T., Ishii, H. and Takizawa, H. (2013) Virus-Induced Exacerbations in Asthma and COPD. Frontiers in Microbiology, 4, 293. https://doi.org/10.3389/fmicb.2013.00293

[74] Yang, I.A., Clarke, M.S., Sim, E.H. and Fong, K.M. (2012) Inhaled Corticosteroids for Stable Chronic Obstructive Pulmonary Disease. Cochrane Database of Systematic Reviews, No. 7, CD002991. https://doi.org/10.1002/14651858.CD002991.pub3

[75] Contoli, M., Pauletti, A., Rossi, M.R., et al. (2017) Long-Term Effects of Inhaled Corticosteroids on Sputum Bacterial and Viral Loads in COPD. European Respira- 
tory Journal, 50, Article ID: 1700451. https://doi.org/10.1183/13993003.00451-2017

[76] Schaller, T., Hirschbühl, K., Burkhardt, K., et al. (2020) Postmortem Examination of Patients with COVID-19. JAMA, 323, 2518-2520.

https://doi.org/10.1001/jama.2020.8907

[77] van den Brand, J.M.A., Smits, S.L. and Haagmans, B.L. (2015) Pathogenesis of Middle East Respiratory Syndrome Coronavirus. Journal of Pathology, 235, 175-184. https://doi.org/10.1002/path.4458

[78] Ito, K., Ohmagari, N., Mikami, A. and Sugiura, W. (2020) Major Ongoing Clinical Trials for COVID-19 Treatment and Studies Currently Being Conducted or Scheduled in Japan. Global Health \& Medicine, 2, 96-101. https://doi.org/10.35772/ghm.2020.01034

[79] Gralinski, L.E., Sheahan, T.P., Morrison, T.E., et al. (2018) Complement Activation Contributes to Severe Acute Respiratory Syndrome Coronavirus Pathogenesis. mBio, 9, e01753-18. https://doi.org/10.1128/mBio.01753-18

[80] Zhong, J., Tang, J., Ye, C. Dong, L. (2020) The Immunology of COVID-19: Is Immune Modulation an Option for Treatment? The Lancet Rheumatology, 2, E428-E436. https://doi.org/10.1016/S2665-9913(20)30120-X

[81] Mehta, P., McAuley, D.F., Brown, M., Sanchez, E., Tattersall, R.S. and Manson, J.J. (2020) COVID-19: Consider Cytokine Storm Syndromes and Immunosuppression. The Lancet, 395, 1033-1034. https://doi.org/10.1016/S0140-6736(20)30628-0

[82] Nakajima, K., Ogawa, F., Sakai, K., et al. (2020) A Case of Coronavirus Disease 2019 Treated with Ciclesonide. Mayo Clinic Proceedings, 95, 1296-1297. https://doi.org/10.1016/j.mayocp.2020.04.007

[83] Ito, K., Yokoyama, T., Oguri, A., et al. (2020) Case Report: A Case of COVID-19 Pneumonia That Did Not Worsen and Was Relieved by Early Administration of Favipiravir and Ciclesonide.

[84] (2020) A Trial of Ciclesonide in Adults with Mild COVID-19. Clinical-Trials.gov. https://clinicaltrials.gov/ct2/show/NCT04330586

[85] Iwabuchi, K., Yoshie, K., Kurakami, Y., Takahashi, K., Kato, Y. and Morishima, T. (2020) Therapeutic Potential of Ciclesonide Inhalation for COVID-19 Pneumonia: Report of Three Cases. Journal of Infection and Chemotherapy, 26, 625-632. https://doi.org/10.1016/j.jiac.2020.04.007

[86] Al-Dorzi, H.M., al Harbi, S.A. and Arabi, Y.M. (2014) Antibiotic Therapy of Pneumonia in the Obese Patient: Dosing and Delivery. Current Opinion in Infectious Diseases, 27, 165-173. https://doi.org/10.1097/QCO.0000000000000045

[87] Iannella, H., Luna, C. and Waterer, G. (2016) Inhaled Corticosteroids and the Increased Risk of Pneumonia: What's New? A 2015 Updated Review. Therapeutic Advances in Respiratory Disease, 10, 235-255. https://doi.org/10.1177/1753465816630208

[88] Spagnolo, P., Balestro, E., Aliberti, S., et al. (2020) Pulmonary Fibrosis Secondary to COVID-19: A Call to Arms? The Lancet Respiratory Medicine, 8, 750-752. https://doi.org/10.1016/S2213-2600(20)30222-8

[89] Stein, R., Sherril, D.L., Morgan, W.J., Holberg, C.J., Halonen, M., Taussig, L., Wright, A. and Martinez, F.D. (1999) Respiratory Syncytial Virus in Early Life and Risk of Wheeze and Allergy by Age 13 Years. The Lancet, 354, 541-545. https://www.sciencedirect.com/science/article/pii/S0140673698103215 https://doi.org/10.1016/S0140-6736(98)10321-5

[90] Woods, J.A., Wheeler, J.S., Finch, C.K. and Pinner, N.A. (2014) Corticosteroids in the Treatment of Acute Exacerbations of Chronic Obstructive Pulmonary Disease. International Journal of COPD, 9, 421-430. https://doi.org/10.2147/COPD.S51012 